
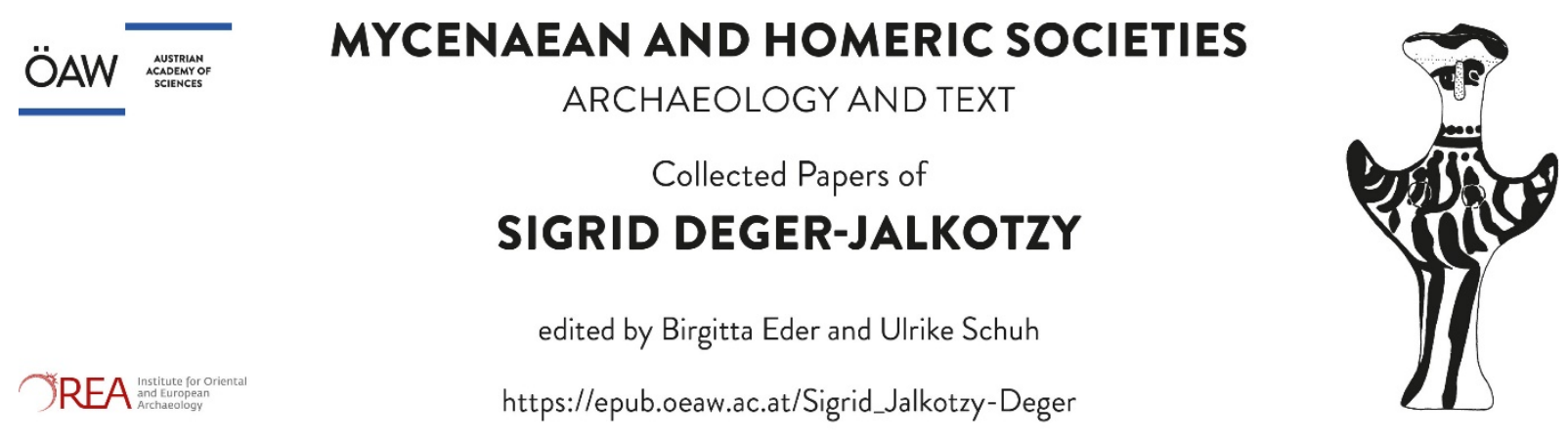

58.

S. Deger-Jalkotzy

\title{
Laudatio für Malcolm Wiener
}

Almanach der Österreichischen Akademie der Wissenschaften 154, 2004, 144-147

(c) Verlag der ÖAW

mit freundlicher Genehmigung / with kind permission

Dieses Dokument darf ausschließlich für wissenschaftliche Zwecke genutzt werden (Lizenz CC BY-NC-ND), gewerbliche Nutzung wird urheberrechtlich verfolgt.

This document is for scientific use only (license CC BY-NC-ND), commercial use of copyrighted material will be prosecuted. 


\section{ÖSTERREICHISCHE}

AKADEMIE DER WISSENSCHAFTEN

\section{A L M A N A C H $2003 / 2004$ \\ 154. JAHRGANG}

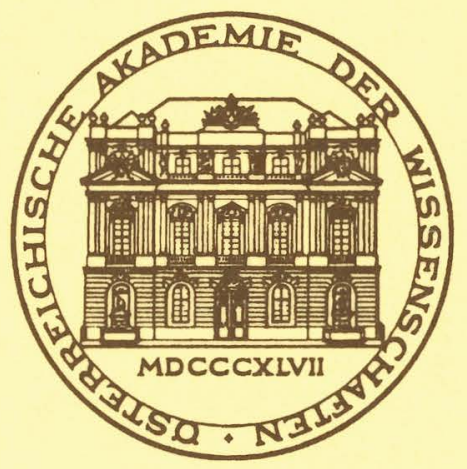

VERLAG DER

ÖSTERREICHISCHEN AKADEMIE DER WISSENSCHAFTEN WIEN 2004 


\section{ÖSTERREICHISCHE AKADEMIE DER WISSENSCHAFTEN}

\section{A L M A N A C H $2003 / 2004$ \\ 154. JAHRGANG}

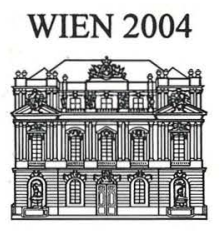

VERLAG DER

ÖSTERREICHISCHEN AKADEMIE DER WISSENSCHAFTEN A-1010 WIEN, DR.-IGNAZ-SEIPEL-PLATZ 2 


\section{Präsidium}

\section{Präsident der Akademie}

und Vorsitzender der mathematisch-naturwissenschaftlichen Klasse

Herbert Mang

o. Professor der Elastizitäts- und Festigkeitslehre

an der Technischen Universität Wien,

1130 Wien, Tolstojgasse $5 / 10$

\section{Vizepräsident der Akademie}

und Vorsitzender der philosophisch-historischen Klasse

\section{Herbert Matis}

o. Professor der Wirtschafts- und Sozialgeschichte

an der Wirtschaftsuniversität Wien,

1180 Wien, Gregor-Mendel-Straße 5/5

\section{Generalsekretär der Akademie} und Sekretär der philosophisch-historischen Klasse

Herwig Friesinger

o. Professor der Ur- und Frühgeschichte

an der Universität Wien,

3424 Zeiselmauer, Bahnhofplatz 6

\section{Sekretär}

der mathematisch-naturwissenschaftlichen Klasse

\section{Georg Stingl}

o. Professor der Dermatologie an der Universität Wien,

1070 Wien, St.-Ulrichs-Platz 2/7 


\section{Redaktioneller Hinweis}

Der vorliegende 154. Jahrgang umfasst den Berichtszeitraum Oktober 2003 bis September 2004 bzw. Juni 2003 bis Mai 2004 für das Publikationsverzeichnis der Mitglieder.

Die verwendeten personenbezogenen Ausdrücke beziehen sich gleichermaßen auf Frauen und Männer.

Redaktion: Johann Felfernig, Ingrid Weichselbaum

Die verwendeten Papiersorten sind aus chlorfrei gebleichtem Zellstoff hergestellt, frei von säurebildenden Bestandteilen und alterungsbeständig.

Alle Rechte vorbehalten

ISBN 3-7001-3342-1 geb.,

ISBN 3-7001-3343-X brosch.

ISSN $0378-8644$

Copyright (C) 2004 by

Österreichische Akademie der Wissenschaften

Wien

Druck: Grasl Druck \& Neue Medien, 2540 Bad Vöslau

http://hw.oeaw.ac.at/3342-1 geb.

http://hw.oeaw.ac.at/3343-X brosch.

http://verlag.oeaw.ac.at 


\section{Inhalt}

Seite

Präsidium

\section{Mitglieder}

Verzeichnis der Mitglieder der Österreichischen Akademie der Wissenschaften

Mitglieder der mathematisch-naturwissenschaftlichen Klasse

Wirkliche Mitglieder . . . . . . . . . . . . . . . . 13

Ehrenmitglieder. . . . . . . . . . . . . . . . . . 28

Korrespondierende Mitglieder im Inland . . . . . . . . . 30

Korrespondierende Mitglieder im Ausland . . . . . . . . . 39

Mitglieder der philosophisch-historischen Klasse

Wirkliche Mitglieder . . . . . . . . . . . . . . . . . . . 67

Ehrenmitglieder. . . . . . . . . . . . . . . . . . 80

Korrespondierende Mitglieder im Inland . . . . . . . . . . 81

Korrespondierende Mitglieder im Ausland . . . . . . . . . 91

Veränderungen seit Gründung der Akademie . . . . . . . . . 115

Verstorbene Mitglieder der Akademie im Jahre 2003/2004

(Oktober-September) .

Neu gewählte Mitglieder der Akademie im Jahre 2004

Mathematisch-naturwissenschaftliche Klasse .

Philosophisch-historische Klasse

\section{Publikationen}

Verzeichnis der im Jahre 2003/2004 (Juni-Mai) erschienenen Publikationen der wirklichen Mitglieder und der korrespondierenden Mitglieder im Inland

Mathematisch-naturwissenschaftliche Klasse .

Philosophisch-historische Klasse 
Verzeichnis der im Jahre 2003/2004 (Oktober-September) erschienenen

Publikationen der Österreichischen Akademie der Wissenschaften

Gesamtakademie

Mathematisch-naturwissenschaftliche Klasse . . . . . . . . . 240

Philosophisch-historische Klasse . . . . . . . . . . . . 241

Die Feierliche Sitzung am 12. Mai 2004

Begrüßung durch den Präsidenten der Akademie Herbert Mang . . . 251

Ansprache des Bundespräsidenten Thomas Klestil. . . . . . . . 255

Bericht des Vizepräsidenten Herbert Matis . . . . . . . . . . 261

Bericht des Generalsekretärs Herwig Friesinger . . . . . . . . . 267

Bericht des Sekretärs Georg Stingl . . . . . . . . . . . . 277

Verleihung der Medaille „Bene merito“ . . . . . . . . . . . . 287

Ansprache des Präsidenten . . . . . . . . . . . . . . . 289

Abschiedsworte zum Ableben von Frau Hofrat Dr. Margarete Bieder . . 297

Verleihung der Medaille „Bene merito“ und der Akademie-Preise . . . 303

Präsidium und zentrale Verwaltung der Akademie

Präsidium . . . . . . . . . . . . . . . . . . . . 307

Präsidialsekretariat . . . . . . . . . . . . . . . . . 307

Generalsekretariat . . . . . . . . . . . . . . . . . . 308

Rechts- und Personalangelegenheiten. . . . . . . . . . . . 308

Verwaltungsstellen der beiden Klassen . . . . . . . . . . . 308

Rechnungswesen und EDV-Belange . . . . . . . . . . . . . . 309

Bibliothek und Archiv . . . . . . . . . . . . . . . . . . . . . 310

Bauwesen . . . . . . . . . . . . . . . . . . . . 310

Hausbetriebsstelle . . . . . . . . . . . . . . . . 310

Auslandsbeziehungen . . . . . . . . . . . . . . . . 311

Stipendien- und Preisangelegenheiten . . . . . . . . . . . . 311

Verlag . . . . . . . . . . . . . . . . . . . . . 311

Akademie-Rechenzentrum . . . . . . . . . . . . . . . 312

Betriebsrat . . . . . . . . . . . . . . . . . . . . 312

Sicherheitsvertrauenspersonen . . . . . . . . . . . . . 313 


\section{Kommissionen der Akademie \\ Zusammensetzung und Mitarbeiter}

Gemeinsame Kommissionen

Wissenschaftliche Kommissionen

Geschichte der Naturwissenschaften, Mathematik und Medizin . . . 315

Prähistorische K. . . . . . . . . . . . . . . . . . . . . 316

Quartärforschung . . . . . . . . . . . . . . . . . 316

K. für die wissenschaftliche Zusammenarbeit mit Dienststellen des BM für Landesverteidigung . . . . . . . . . . . . . 317

Historische Pressedokumentation . . . . . . . . . . . . 317

Interdisziplinäre ökologische Studien . . . . . . . . . . . 318

Verwaltungskommissionen

Planungsk. . . . . . . . . . . . . . . . . . . . 318

Akademiebibliothek . . . . . . . . . . . . . . . . . 319

Rechnungskontrollk. . . . . . . . . . . . . . . . . . . 319

Verlagsk. . . . . . . . . . . . . . . . . . . . . 319

Kommissionen der math.-nat. Klasse

Wissenschaftliche Kommissionen

Astronomie . . . . . . . . . . . . . . . . . . 321

Reinhaltung der Luft . . . . . . . . . . . . . . . . 321

Geophysikalische Forschungen . . . . . . . . . . . . . . . . . 322

Grundlagen der Mineralrohstoffforschung . . . . . . . . . 322

Paläontologische und stratigraphische Erforschung Österreichs . . . . 322

Wissenschaftliche Visualisierung . . . . . . . . . . . . 323

Verwaltungskommission

Budgetk. . . . . . . . . . . . . . . . . . . . . 323

Verleihung des Erwin-Schrödinger-Preises . . . . . . . . . 324

Verleihung des Fritz-Pregl-Preises . . . . . . . . . . . . 324

Verleihung des Alois-Sonnleitner-Preises . . . . . . . . . . 325

Verleihung des Othenio-Abel-Preises . . . . . . . . . . . . . 325

Verleihung des Edmund-und-Rosa-Hlawka-Preises . . . . . . . . 325

Verleihung des Ignaz-L.-Lieben-Preises . . . . . . . . . . 326 
Kommissionen der phil.-hist. Klasse

Kultur und Geschichte Österreichs und des Donauraumes

Historische K. . . . . . . . . . . . . . . . . . . 327

Geschichte der Habsburgermonarchie . . . . . . . . . . . 327

Schrift- und Buchwesen des Mittelalters . . . . . . . . . . 328

Literaturwissenschaft . . . . . . . . . . . . . . . . 329

Kulturwissenschaften und Theatergeschichte . . . . . . . . 330

Rechtsgeschichte Österreichs . . . . . . . . . . . . . . . 331

Migrations- und Integrationsforschung. . . . . . . . . . . . 339

Kultur und Sozialgeschichte des römischen und byzantinischen

Reiches, des Mittelmeerraumes und des Nahen Ostens

Antike Rechtsgeschichte . . . . . . . . . . . . . . . 331

Byzantinistik . . . . . . . . . . . . . . . . . . 332

Tabula Imperii Byzantini . . . . . . . . . . . . . . . 332

Numismatische K. . . . . . . . . . . . . . . . . . . . . . 333

Ägypten und Levante . . . . . . . . . . . . . . . . . . . . 333

Mykenische K. . . . . . . . . . . . . . . . . . . . . . 334

Balkan-K. . . . . . . . . . . . . . . . . . . . 334

Kleinasiatische K. . . . . . . . . . . . . . . . . . 335

Allgemeine Sprachwissenschaft

Linguistik und Kommunikationsforschung . . . . . . . . . 336

Musik- und Kunstforschung

Kunstgeschichte. . . . . . . . . . . . . . . . . . 336

Musikforschung . . . . . . . . . . . . . . . . . . 337

Lexikographie und Editionen

Textwörterbuch der Fackel . . . . . . . . . . . . . . . . . . . 329

Herausgabe des Corpus der lateinischen Kirchenväter (CSEL) . . . 337

Antike Literatur und lateinische Tradition . . . . . . . . . . 338

Sozialanthropologie . . . . . . . . . . . . . . . . 338

Verwaltungskommissionen

Budgetk. . . . . . . . . . . . . . . . . . . . . 339

Verleihung des Wilhelm-Hartel-Preises . . . . . . . . . . 340

Verleihung des Figdor-Preises . . . . . . . . . . . . . 340 
Moritz-Csáky-Widmung . . . . . . . . . . . . . . . . 341

Richard-Georg-Plaschka-Legat . . . . . . . . . . . . . . 341

Dr.-Friedrich-Teßmann-Sammlung . . . . . . . . . . . . 341

Publikationsk. . . . . . . . . . . . . . . . . . . 342

Projekte der phil.-hist. Klasse . . . . . . . . . . . . . . . . . 342

\section{Unternehmungen der Akademie \\ Zusammensetzung und Mitarbeiter}

Gesamtakademie

Galerie der Forschung . . . . . . . . . . . . . . . . . . 343

Philosophisch-historische Klasse

AAC - Austrian Academy Corpus .

Institute und Forschungsstellen der Akademie

Zusammensetzung der Kuratorien und Beiräte, Mitarbeiter

Institute der Gesamtakademie

Konrad-Lorenz-I. für Vergleichende Verhaltensforschung . . . . 345

Technikfolgen-Abschätzung . . . . . . . . . . . . . . 346

Phonogrammarchiv. . . . . . . . . . . . . . . . . 347

Österreichisches Biographisches Lexikon und biographische

Dokumentation . . . . . . . . . . . . . . . . . 348

Schallforschung . . . . . . . . . . . . . . . . . . . . 349

Institute der math.-nat. Klasse

Stefan-Meyer-I. für subatomare Physik . . . . . . . . . . . 350

Hochenergiephysik . . . . . . . . . . . . . . . . . 351

Limnologie . . . . . . . . . . . . . . . . . . . 354

Erich-Schmid-I. für Materialwissenschaft . . . . . . . . . . 356

Weltraumforschung . . . . . . . . . . . . . . . . . 358

Biophysik und Röntgenstrukturforschung . . . . . . . . . . . . 361

Biomedizinische Alternsforschung . . . . . . . . . . . . . 362

Johann Radon I. for Computational and Applied Mathematics . . . 364

Quantenoptik und Quanteninformation. . . . . . . . . . . 365

Molekulare Biotechnologie . . . . . . . . . . . . . . 366 
IMP-IMBA Genome Research Center .

Molekulare Medizin

Gregor-Mendel-I. für Molekulare Pflanzenbiologie $\mathrm{GmbH}$

Institute der phil.-hist. Klasse

Realienkunde des Mittelalters und der frühen Neuzeit . . . . . . 368

Demographie . . . . . . . . . . . . . . . . . . 369

Stadt- und Regionalforschung . . . . . . . . . . . . . 371

Kultur- und Geistesgeschichte Asiens . . . . . . . . . . . 372

Österreichische Dialekt- und Namenlexika . . . . . . . . . . 373

Kulturgeschichte der Antike . . . . . . . . . . . . . . . . . 374

Iranistik . . . . . . . . . . . . . . . . . . . 375

Mittelalterforschung . . . . . . . . . . . . . . . . 376

Europäische Integrationsforschung . . . . . . . . . . . 378

Forschungsstellen

Integrierte Sensorsysteme . . . . . . . . . . . . . . . 379

Europäisches Schadenersatzrecht . . . . . . . . . . . . 379

Forschungsprogramme

Man and Biosphere (MAB) . . . . . . . . . . . . . . 381

Hydrologie Österreichs . . . . . . . . . . . . . . . . . . . . . 381

International Geoscience Programme (IGCP). . . . . . . . . 382

Global Change Program . . . . . . . . . . . . . . . . . 382

International Strategy for Disaster Reduction (ISDR) . . . . . . 383

Geophysik der Erdkruste . . . . . . . . . . . . . . . . . . 383

Alpenforschung . . . . . . . . . . . . . . . . . . 384

Werkstoffkompetenzzentrum - Leoben Forschungsges.m.b.H. . . . 385

Stipendienprogramme

Doktorandenprogramme . . . . . . . . . . . . . . . 386

APART - Austrian Programme for Advanced Research and Technology 387

Max-Kade-Stipendien . . . . . . . . . . . . . . . . 324

Einrichtungen bei der ÖAW

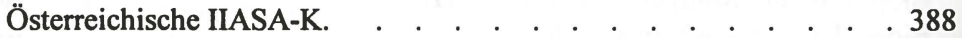

Kernfusionsforschung . . . . . . . . . . . . . . . . . . 389

Entwicklungsfragen . . . . . . . . . . . . . . . . 389

Rekombinante Gen-Technik . . . . . . . . . . . . . . 392 
Kuratorium der Akademie für das Historische Institut beim

Österreichischen Kulturforum in Rom . . . . . . . . . . 392

Spezialforschungsbereich SCIEM 2000 . . . . . . . . . . . . 393

In Verbindung mit der ÖAW

Ständiges Sekretariat der International Federation of Automatic

Control (IFAC) . . . . . . . . . . . . . . . . . . . . . 394

Int. Lehrgang in Limnologie für Postgraduierte aus Entwicklungsländern 394

Arbeitsgemeinschaft für Kartographische Ortsnamenkunde (AKO) . . 394

Hannes Androsch Stiftung bei der ÖAW . . . . . . . . . . 395

\section{Delegationen}

Delegationen im Rahmen des ICSU. . . . . . . . . . . . . . 396

Sonstige Delegationen . . . . . . . . . . . . . . . . . . 401

Liste der von der Akademie zu vergebenden Preise $\quad . \quad . \quad . \quad . \quad . \quad .409$

Gesellschaft der Freunde der ÖAW

Übersicht der Sitzungen der Akademie im Jahre 2004 . . . . . . . 416

\section{Nachrufe}

Auf Mitglieder der math.-nat. Klasse

w. M. Anton Pischinger . . . . . . . . . . . . . . . 421

k. M. Peter Safar . . . . . . . . . . . . . . . . . . . . . 4427

k. M. André Guinier . . . . . . . . . . . . . . . . 437

k. M. Ernst Friedrich Lüscher . . . . . . . . . . . . . 445

Auf Mitglieder der phil.-hist. Klasse

w. M. Margret Dietrich . . . . . . . . . . . . . . . 453

w. M. Mario Wandruszka . . . . . . . . . . . . . . 461

k. M. Olivier Reverdin . . . . . . . . . . . . . . . . 467

k. M. Robert Braidwood . . . . . . . . . . . . . . . 473

k. M. Johannes Duft . . . . . . . . . . . . . . . . 479

k. M. Günter Kahle . . . . . . . . . . . . . . . . . 487

k. M. Friedrich Prinz . . . . . . . . . . . . . . . . 495 
k. M. Franciscus Bernardus Jacobus Kuiper . . . . . . . . . 503

k. M. Andreas Tietze . . . . . . . . . . . . . . . . . . . 511

k. M. Günther Beitzke. . . . . . . . . . . . . . . . 517

Personenregister . . . . . . . . . . . . . . . . . . 523 
profiliert, ja die Anerkennung der Frühneuzeit als deutlich ab. grenzbares Epochenfach ist ganz wesentlich mit Schulzes Werk verbunden. Als zweiter bedeutender Forschungsschwerpunkt gelten Schulzes Beiträge zur Geschichte der deutschen Geschichts. wissenschaft im 20. Jahrhundert. Schulze hat weit über sein Fach hinaus Anerkennung gefunden mit der Verleihung des Leibniz. Preises 1996 und der Wahl zum Präsidenten des Wissenschafts. rates der BRD für die Funktionsperiode 1998-2001.

GERALD STOURZH

Wiener, Malcolm Hewitt: Geboren am 3. Juli 1935 in Tsingtao,

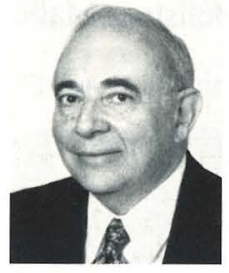
China, wo sein Vater als Richter am Internationalen Handelsgerichtshof tätig war, kam Malcolm Wiener 1939 in die USA und erhielt dort seine Ausbildung. An der Harvard University schloss er sein erstes Studium in den Wirtschaftswissenschaften 1957 ab. Nach dem Militärdienst bei der USNavy, der ihn zum ersten Mal in die Ägäis und nach Zypern führte, studierte er an der Harvard Law School, wo er auch zum Dr. juris promoviert wurde. In den darauf folgenden Jahren machte er Karriere als Jurist und im Investment Management. Darüber hinaus diente er dem US-Außenministerium als Rechtsberater u. a. im Zusammenhang mit illegalem Antiquitätenhandel. Ebenso war er Berater des Archaeological Institute of America. Nachdem Malcolm Wiener bereits neben seinen wirtschaftswissenschaftlichen und juristischen Studien Lehrveranstaltungen in den Klassischen Altertumswissenschaften belegt hatte, trat dieses Interesse mit der Zeit immer mehr in den Vordergrund. Von 1981 an widmete er sich schließlich neben verschiedenen öffentlichen Verpflichtungen vorwiegend der althistorischen Forschung und machte die frühen Hochkulturen Griechenlands zu seinem Forschungsschwerpunkt. Es gehört zu Malcolm Wieners bleiben- 
den Verdiensten, als Erster auf die ökonomischen Aspekte der so genannten „Minoischen Seeherrschaft“ hingewiesen zu haben. Für den engen Zusammenhang zwischen der enormen kulturellen Ausstrahlung der minoischen Kultur und ihrer ökonomischen Überlegenheit gegenüber den anderen Regionen der Ägäis und insbesondere gegenüber der inzipienten mykenischen Kultur erfand er den Begriff „Versailles-Effekt", der seither seinen festen Platz in der Erforschung des 2. Jahrtausends v. Chr. behauptet. Ebenso machte er in den beiden letzten Jahrzehnten des vorigen Jahrhunderts historische Fragestellungen für die Forschungsarbeit an der minoischen und der mykenischen Kultur, die damals - namentlich in den angelsächsischen Ländern - weitgehend eine Domäne der Prähistoriker war und sich vorwiegend auf lokale Entwicklungsabläufe der ägäischen Kulturen konzentrierte, wieder hoffähig. Seine Arbeiten über die Natur und Kontrolle des minoischen Handels, wie auch über die Beziehungen des minoischen Kreta und des mykenischen Griechenland zu den Hochkulturen des Vorderen Orients und Ägyptens leiteten geradezu einen „Boom“ wirtschaftsgeschichtlicher Untersuchungen zur minoischen und mykenischen Periode Griechenlands ein. Seine 1989 erschienene Studie „The Isles of Crete?" wird bis heute an vielen Universitäten als Einführung in die Geschichte der bronzezeitlichen Ägäis empfohlen. Ebenso gehört sein Beitrag über die Bedeutung von Siegeln und Siegelabdrücken für das Verständnis der minoischen Administration und Kontrolle von Wirtschaftsvorgängen (1990) zu den grundlegenden Arbeiten über dieses Thema. Seit einigen Jahren konzentriert sich Malcolm Wiener auf die Chronologie der Spätbronzezeit in der Ägäis. Neuere naturwissenschaftliche Datierungsmethoden weichen weit von der traditionellen, auf kulturellen Querverbindungen zwischen Kreta und dem Neuen Reich Ägyptens basierenden Chronologie der Jüngeren Palastzeit Kretas und der frühen mykenischen Periode $\mathrm{ab}$ und haben zu einem heftigen internationalen Gelehrtenstreit geführt. Wieners Bemühungen um eine Klärung dieser für Historiker so wichtigen Frage trifft sich 
direkt mit den Interessen des an der Österreichischen Akademie der Wissenschaften beheimateten SFB „The Synchronisation of Civilisations in the Eastern Mediterranean in the Second Millennium B.C." (SCIEM 2000), an dessen Veranstaltungs- und Publika. tionstätigkeiten er lebhaften Anteil nimmt. Er gehört zu den ganz wenigen Gelehrten, die sowohl die naturwissenschaftlichen Metho. den der Radiokarbondatierung, der Dendrochronologie und der Analyse von Grönlandeis-Bohrkernen verstehen und nachvollziehen können, als auch die altorientalischen astronomischen Texte heranziehen und die archäologische Evidenz für die Beziehungen zwischen Ägypten, dem Vorderen Orient und den ägäischen Kulturen beherrschen. Nicht wenige Kollegen brachte er mit dieser weiten fachlichen Kompetenz in Verlegenheit. Bereits neun Beiträge zu diesem Thema hat er in den letzten fünf Jahren geschrieben, darunter ein umfassendes Manuskript zur absoluten Chronologie des 14. Jh. v. Chr. Der SFB SCIEM 2000 darf sich rühmen, die Forschungen Malcolm Wieners in seine Arbeit zu integrieren. Abgesehen von seinen Publikationen zur ägäischen Frühgeschichte widmete Malcolm Wiener auch anderen Themen sein Interesse, wie etwa sein Artikel „Picasso and the Cuban Missile Crisis“ (Apollo, Oktober 2001) verrät. Eine Würdigung Malcolm Wieners wäre unvollständig ohne die Erwähnung seines Mäzenatentums. 1982 stiftete er das Institute for Aegean Prehistory in New York, das seine Aufgabe durch die finanzielle Unterstützung von Ausgrabungen und Publikationen zur ägäischen Ur- und Frühgeschichte wahrnimmt und dem ein internationales Kuratorium vorsteht. Weiters gründete er in Ostkreta ein archäologisches Studienzentrum, an der American School of Classical Studies in Athen das Wiener Laboratory für naturwissenschaftliche Untersuchungsmethoden archäologischer Funde, und gemeinsam mit seiner Ehefrau, Dr. Carolyn Talbot Seely Wiener das Malcolm und Carolyn Wiener Laboratory for Aegean and Near Eastern Dendrochronology an der Cornell University. Malcolm Wiener hat somit als Forscher und als Förderer Großes für die Weiterentwicklung der Altertumswissenschaften geleistet. 
Ohne ihn wäre vieles nicht in die Wege geleitet und vieles nicht vollendet worden.

Ausgewählte Publikationen:

Trade and Rule in Palatial Crete. In: R. Hägg, N. Marinatos (Eds.), The Function of the Minoan Palaces. Stockholm 1987, 261-266.

Round Table Comments. In: T. G. Palaima (Ed.), Aegean Seals, Sealings and Administration. Liège 1990, 236-239.

Time Out: The Current Impasse in Bronze Age Archaeological Dating. In: K. P. Foster, R. Laffineur (Eds.), METRON, Measuring the Aegean Bronze Age. Liège - Austin 2003, 363-399. The Absolute Chronology of Late Helladic IIIA 2 Revisited. Annual of the British School at Athens, 98 (2003), 239-250.

Times Change: The Current State of the Debate in Old World Chronology. In: Proceedings of the $2^{\text {nd }}$ Euro Conference of SCIEM 2000, Vienna 2003 (im Druck).

SIGRID JALKOTZY-DEGER 